\title{
TRANSFERÊNCIA DE CALOR EM LEITO MÓVEL DE XISTO
}

\author{
C. C. MORATORI ${ }^{1 *}$, A. C. L. LISBÔA ${ }^{1}$ \\ ${ }^{1}$ Universidade Estadual de Campinas, Faculdade de Engenharia Química \\ "e-mail: ccmoratori@yahoo.com.br
}

\begin{abstract}
RESUMO
O xisto é uma rocha compacta de origem sedimentar, formada por componentes orgânicos complexos distribuídos numa matriz mineral. A matéria orgânica, chamada querogênio, é liberada sob aquecimento em temperaturas acima de $350{ }^{\circ} \mathrm{C}$ em retortas. $\mathrm{O}$ óleo obtido quando refinado produz destilados com as mesmas características encontradas nos de petróleo, sendo considerado uma fonte de energia alternativa. Para o projeto dos reatores de leito móvel para pirólise é necessário o conhecimento de várias etapas, inclusive sobre a transferência de calor entre as partículas de xisto e a corrente gasosa. Estas trocas podem ser avaliadas por modelos matemáticos solucionados por métodos numéricos. Neste trabalho foram construídos modelos que descrevem os perfis de temperatura no processo em que as partículas de xisto apresentam gradiente de temperatura em seu interior devido ao seu tamanho. As simulações foram realizadas por programas em linguagem FORTRAN.
\end{abstract}

\section{INTRODUÇÃO}

Pela facilidade de seu uso e pela capacidade de atender às necessidades de seus usuários, o petróleo tornou-se responsável por mais de $40 \%$ da energia consumida no mundo (IEA, 2009).

Como o suprimento de petróleo é incerto e esgotável, o Brasil iniciou estudos para o aproveitamento de xisto por ser detentor da segunda maior reserva mundial deste mineral. A exploração se desenvolveu na região de São Mateus do Sul (PR), onde há uma jazida, pertencente à Formação Irati parte integrante da coluna de sedimentos paleozóicos de idade Permiana - que apresentava condições favoráveis (TONEL et al., 2004).

A exploração dessa reserva vem sendo executada pela SIX (Unidade de Negócios da Industrialização do Xisto), que é uma unidade da Petrobras criada em 1954 para desenvolver estudos sobre o aproveitamento de xisto. Essa unidade possui capacidade instalada de $7 \mathrm{mil}$ toneladas de xisto por dia (PETROBRAS, 2014). Para tanto foi desenvolvido a partir de 1958, pelos técnicos da Petrobras, um processo de transformação de xisto que recebeu o nome de Petrosix, que emprega um reator em leito móvel.

São importantes estudos sobre as etapas da pirólise, tais como: cinética de desvolatilização - determinação da energia de ativação e constante da taxa de reação - e investigações dos fenômenos de transferência de calor e massa. Tais conhecimentos constituem aspectos fundamentais quando se deseja aumentar a eficiência de processos de pirólise ou projetar novos reatores para a retortagem (ALMEIDA, 2005).

\section{PROCESSO PETROSIX}

O xisto, depois de minerado a "céu aberto", vai para um britador no qual as partículas são reduzidas a um tamanho entre $0,006 \mathrm{~m}$ a $0,07 \mathrm{~m}$. Essas partículas são 
peneiradas e aquelas fora da especificação ou retornam ao britador (grossas), ou retornam à mina (finas). As demais são distribuídas no topo do reator, de onde se movem lentamente por gravidade, em um leito compactado, para a parte inferior da retorta.

O calor para a pirólise é fornecido por uma corrente gasosa de elevada temperatura que entra na zona de pirólise e se mistura com uma segunda corrente, injetada na base da retorta, para recuperar o calor do xisto já retortado. Esta corrente gasosa é constituída por aproximadamente $85 \%$ de vapor d'água.

Na retorta há quatro zonas distintas por onde escoam as partículas de xisto. Essas zonas e o processo completo podem ser visualizados na Figura 1. No reator, as partículas de xisto são aquecidas da temperatura ambiente até aproximadamente 773 K. O material orgânico presente no xisto (querogênio) é pirolisado e volatilizado.

Figura 1 - Esquema do processo Petrosix

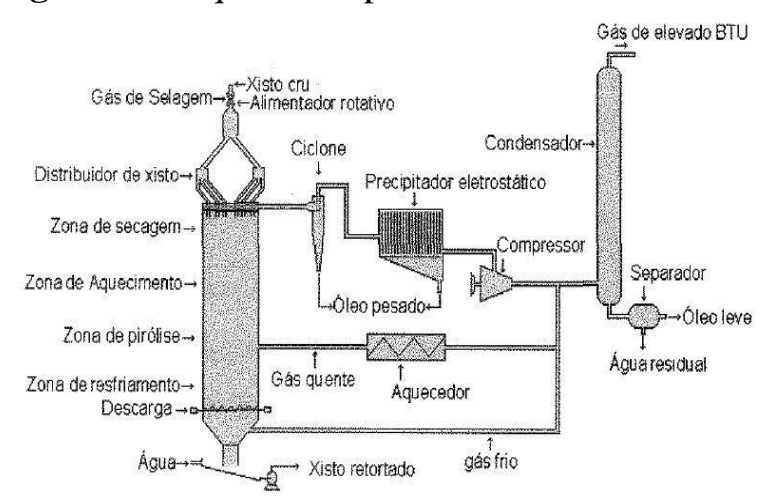

Fonte: Lisbôa (1997)

Os vapores ascendentes, provenientes da zona de pirólise, se condensam logo abaixo do topo da retorta ao entrar em contato com o xisto frio, formando uma neblina de óleo que é arrastada pelo gás. A mistura de fluidos, composta por gases, vapor d'água e neblina de óleo, deixa a retorta e atravessa um conjunto de operações unitárias projetadas para as devidas coletas de pó e óleo, utilizando ciclones e precipitadores eletrostáticos.
Após retortado, o xisto é descarregado em um coletor, misturado com água, resfriado, e transportado para a mina.

\section{BALANÇO DE ENERGIA}

$\mathrm{O}$ reator de pirólise em estudo possui $34 \mathrm{~m}$ de altura e diâmetro de $11 \mathrm{~m}$, porém a região de estudo se restringe a $3 \mathrm{~m}$ de altura compreendendo a zona de secagem, de aquecimento e de pirólise.

$\mathrm{Na}$ modelagem utilizou-se a condutividade térmica de xisto igual a 3,84 J $\mathrm{s}^{-1} \mathrm{~m}^{-1} \mathrm{~K}^{-1}$, segundo Schön (1996). O autor afirma que este valor pode variar dependendo das condições geológicas do local da rocha. Já o coeficiente de transferência de calor foi calculado pela Equação de Levenspiel (1998), Equação 1, adequada para leito fixo, considerando as propriedades do vapor d'água para o gás. Os valores do coeficiente são função da dimensão da partícula.

$$
\bar{h}=\frac{k}{d_{p}}\left(2+1,8 \cdot \operatorname{Re}_{p}^{\frac{1}{2}} \cdot \operatorname{Pr}^{\frac{1}{3}}\right)
$$

Os balanços de energia geraram equações diferenciais ordinárias, que foram resolvidas pelo método de Runge-Kutta Fehlberg. Para a solução das equações diferenciais parciais, utilizou-se o Método Numérico das Linhas de Schiesser (1991, 1994) baseado em diferenças finitas. Para a busca de raízes de funções, decidiu-se pelo uso de um método intervalar ("bracketing method").

Vários balanços térmicos foram feitos e estão apresentados a seguir.

\subsection{Balanço térmico global}

O balanço global de troca térmica numa retorta requer as vazões e temperaturas de entrada e saída do xisto e do gás. A disposição das correntes está apresentada na Figura 2. 
Figura 2 - Representação do leito de pirólise com as variáveis envolvidas no balanço de energia

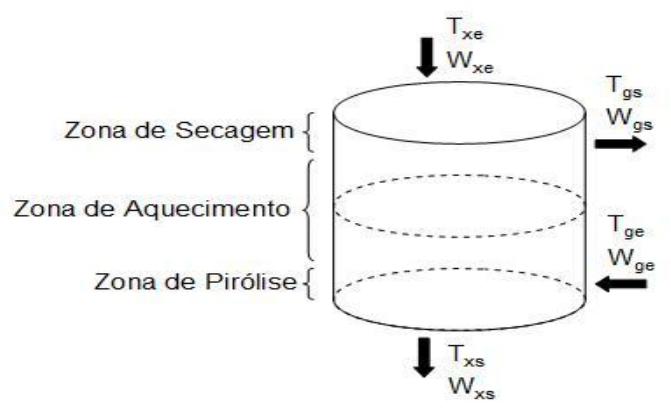

Fonte: Moratori (2014)

Para o xisto adotou-se uma vazão mássica de $65 \mathrm{t} / \mathrm{h}$, calor específico de $1184 \mathrm{~J}$ $\mathrm{kg}^{-1}{ }^{\circ} \mathrm{C}^{-1}$ e temperatura de entrada no topo da retorta de $25{ }^{\circ} \mathrm{C}$. Para o gás, a vazão mássica corresponde a $80 \%$ da vazão mássica do xisto, o calor específico é do vapor d'água, igual a $2050 \mathrm{~J} \mathrm{~kg}^{-1} \mathrm{~K}^{-1}$ e a temperatura da entrada 550 ${ }^{\circ} \mathrm{C}$ na base da retorta.

Como o sistema é adiabático, a quantidade de calor cedida pelo gás é igual àquela recebida pelas partículas de xisto (Equações 2 e 3). As equações possibilitaram validar os modelos construídos.

$$
\begin{aligned}
& Q_{x}=W_{x} \cdot C_{p, x} \cdot\left(T_{x s}-T_{x e}\right) \\
& Q_{g}=W_{g} \cdot C_{p, g} \cdot\left(T_{g s}-T_{g e}\right)
\end{aligned}
$$

\subsection{Balanço térmico na partícula}

Neste modelo matemático foram descritos o gradiente de temperatura no interior das partículas de xisto durante a pirólise na retorta, submetidas a uma corrente gasosa com temperatura fixa $T_{g}$.

A origem do sistema de coordenadas cartesiano $(0,0,0)$ foi colocada no centro da partícula, como mostra a Figura 3.
Figura 3 - Posicionamento do sistema de coordenadas cartesianas na partícula cúbica de xisto (LISBÔA, 1997)

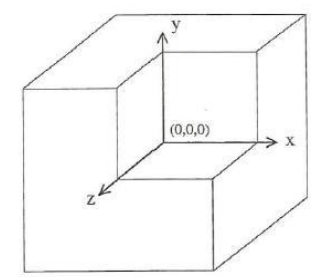

Fonte: Melo (2012)

Supondo valores constantes para as propriedades físicas da partícula de xisto, o balanço de energia pode ser descrito pela equação diferencial parcial (EDP) apresentada na Equação 4. Esta equação considera a variação da temperatura em função do tempo dependente das características do material (representadas pela difusividade térmica $\alpha$ do xisto) e que o gradiente de temperatura está presente em todas as direções da partícula (representado pelo operador Laplaciano estendido a todas as coordenadas do espaço cartesiano).

$$
\frac{\partial T}{\partial t}=\alpha \cdot\left(\frac{\partial^{2} T}{\partial x^{2}}+\frac{\partial^{2} T}{\partial y^{2}}+\frac{\partial^{2} T}{\partial z^{2}}\right)
$$

As condições de contorno das partículas na direção x são mostradas nas Equações 5 e 6. Similares condições existem para as direções y e z. A condição inicial é apresentada na Equação 7.

$$
\begin{array}{ll}
x=0 & \frac{\partial T}{\partial x}=0 \\
x=\frac{L}{2} & -k_{p} \cdot \frac{\partial T}{\partial x}=h \cdot\left(T_{\text {sup }}-T_{g}\right) \\
t=0 & T=T_{0}
\end{array}
$$

A solução numérica das EDPs foi obtida por discretização das dimensões espaciais, gerando um conjunto de equações diferenciais ordinárias - uma para cada ponto de uma 
malha estabelecida na partícula - que é então resolvido.

A Figura 4 apresenta resultados do modelo para uma temperatura de gás constante de $400{ }^{\circ} \mathrm{C}$, considerando partículas paralelepipédicas de dimensões $14 \mathrm{~cm} \times 7 \mathrm{~cm} x 4 \mathrm{~cm}(\mathrm{P} 1), 12 \mathrm{~cm} \times 6 \mathrm{~cm} \times 3 \mathrm{~cm}(\mathrm{P} 2)$ e $9 \mathrm{~cm} \times 5 \mathrm{~cm} \times 3 \mathrm{~cm}(\mathrm{P} 3)$.

Figura 4 - Temperatura no centro e vértice de partículas paralelepipédicas expostas ao gás a 400 ${ }^{\circ} \mathrm{C}$

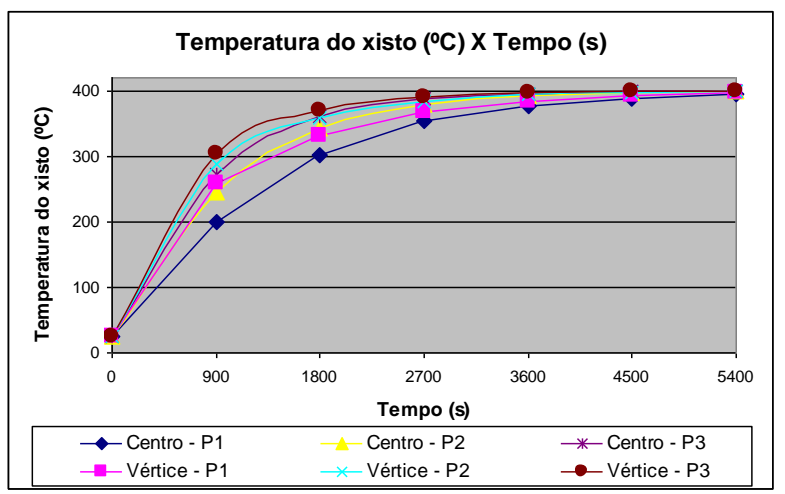

Fonte: Moratori (2014)

Como pode ser verificado na Figura 4, as diferenças de temperatura entre o vértice $\mathrm{e}$ o centro das partículas paralelepipédicas tornam-se mais acentuadas para partículas com maior dimensão e são mais proeminentes no início do processo.

\subsection{Balanço térmico na retorta - Temperatura homogênea nas partículas}

Este modelo matemático considera a troca térmica entre a corrente gasosa e as partículas, e que não há gradiente de temperatura no interior das partículas de xisto. A corrente gasosa está numa temperatura variável $T_{g}$ com calor específico constante $c_{g}$ e a partícula está numa temperatura $T_{\mathrm{x}}$ que varia ao longo do eixo vertical da retorta.

Os balanços de energia do xisto e do gás na retorta ao longo de um comprimento $d z$ são dados pelas Equações 8 e 9. As condições de contorno são dadas pela Equação 10.

$$
\begin{aligned}
& \frac{\partial T_{x}}{\partial z}=\frac{h \cdot a \cdot A_{t}}{W_{x} \cdot c_{c}} \cdot\left(T_{g}-T_{\text {sup }}\right) \\
& \frac{\partial T_{g}}{\partial z}=\frac{h \cdot a \cdot A_{t}}{W_{g} \cdot c_{g}} \cdot\left(T_{g}-T_{\text {sup }}\right) \\
& z=0 \quad T_{x}=T_{x e} \quad T_{g}=T_{g s}
\end{aligned}
$$

Esses balanços de energia são adequados para os casos de umidade de xisto baixa e pequena perda de material pela pirólise (teor de óleo baixo). Caso contrário, são válidos apenas para a região de aquecimento em que não há alterações nas vazões de xisto e gás.

A integração começa pelo topo da retorta, local em que a temperatura do gás de saída $\left(T_{g s}\right)$ é desconhecida, apesar de ser uma condição de contorno (Equação 10). Portanto, a solução requer que essa temperatura seja proposta e variada até que, pela integração até a base da retorta, a correta temperatura do gás na entrada $\left(550^{\circ} \mathrm{C}\right)$ seja atingida.

Os perfis de temperatura do gás e do xisto são mostrados na Figura 5. Resultados semelhantes foram obtidos por Melo e Lisbôa (2012).

Figura 5 - Perfil de temperatura do gás e do xisto (partículas paralelepipédicas sem gradiente térmico) na retorta

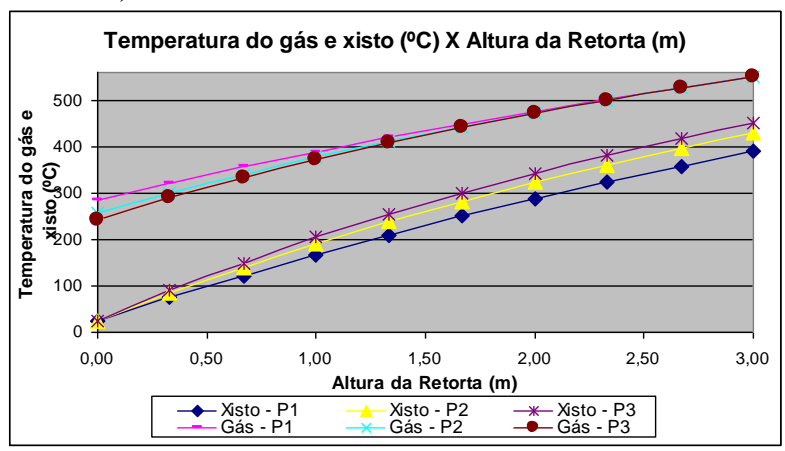

Fonte: Moratori (2014)

Como pode ser visto na Figura 5, a temperatura da saída das partículas de xisto é maior para a menor dimensão da partícula. A 
temperatura do gás na saída é tanto maior quanto maior a dimensão da partícula. Uma partícula maior necessita de maior energia para se aquecer totalmente.

\subsection{Balanço térmico na retorta - Temperatura heterogênea nas partículas}

Neste modelo matemático considerouse gradiente de temperatura nas partículas de xisto. A corrente gasosa está numa temperatura variável $T_{g}$, considerou-se calor específico constante $c_{g}$, e a superfície da partícula está numa temperatura $\overline{T_{\text {sup }}}$ que varia ao longo do eixo vertical da retorta.

As temperaturas em cada ponto interno das partículas de xisto são dadas pela Equação 11, enquanto que a Equação 12 fornece o perfil de temperatura no gás. As condições iniciais estão presentes na Equação 13.

$$
\begin{aligned}
& \frac{\partial T}{\partial t}=\alpha \cdot\left(\frac{\partial^{2} T}{\partial x^{2}}+\frac{\partial^{2} T}{\partial y^{2}}+\frac{\partial^{2} T}{\partial z^{2}}\right) \\
& \frac{\partial T_{g}}{\partial t}=\frac{h \cdot a \cdot A_{t} \cdot v}{W_{g} \cdot c_{g}} \cdot\left(T_{g}-\overline{T_{\text {sup }}}\right) \\
& T=T_{0} \quad T_{g}=T_{g s}
\end{aligned}
$$

Os perfis obtidos das temperaturas médias superficiais do xisto e do gás ao longo do tempo de residência de 90 minutos na retorta estão apresentados na Figura 6.

Figura 6 - Perfil de temperatura do gás e do xisto (partículas paralelepipédicas com gradiente térmico) na retorta

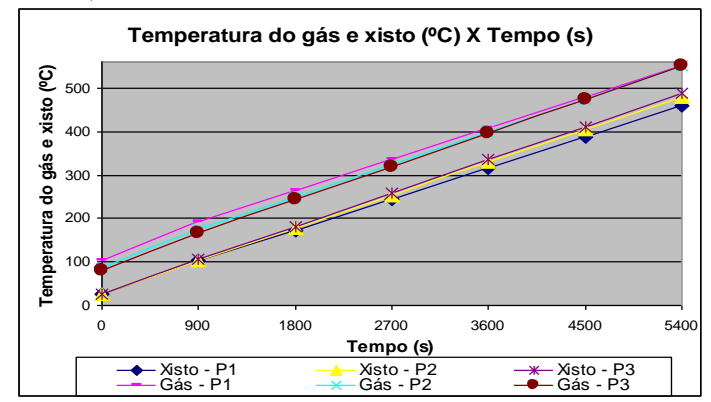

Fonte: Moratori (2014)
Analisando a Figura 6, verifica-se que a temperatura na saída das partículas de xisto é maior para a partícula de menor dimensão, enquanto que a temperatura do gás na saída é maior quando a dimensão da partícula é maior. Resultado análogo ao obtido para partículas de xisto com temperatura homogênea em seu interior.

Partículas menores conseguem se aquecer mais facilmente apresentando temperaturas maiores e, consequentemente, o gás cede maior energia.

\section{CONCLUSÕES}

Os programas redigidos em linguagem FORTRAN permitiram a obtenção de perfis de temperatura das partículas de xisto e do gás ao longo da retorta de pirólise do processo Petrosix. Os valores obtidos se aproximam daqueles do módulo industrial existente, capacitando o modelo como ferramenta para projetos e estudos da pirólise.

Os dados industriais não são apresentados devido a um acordo de sigilo. Valores de vazão e temperaturas foram baseados no processo industrial.

O modelo, bem como a pirólise, é função de parâmetros, tais como: capacidade calorífica, massa específica e condutividade térmica do xisto e do gás, bem como de parâmetros do leito, tais como porosidade.

Para a malha tridimensional da partícula paralelepipédica se confirma a hipótese de que o núcleo aumenta sua temperatura mais lentamente do que a face externa. Conseqüentemente, as temperaturas obtidas no vértice são as maiores da partícula. Além disso, nota-se que as variações da temperatura entre vértice e núcleo se reduzem com a diminuição das dimensões.

\section{NOMENCLATURA}

$\begin{array}{lll}a & \text { Razão: área part. e volume leito } & {\left[\mathrm{m}^{-1}\right]} \\ \alpha & \text { Difusividade térmica } & {\left[\mathrm{m}^{2} \mathrm{~s}^{-1}\right]}\end{array}$ 


$\begin{array}{lll}A_{p, t} & \text { Área total da superf. das part. } & {\left[\mathrm{m}^{2}\right]} \\ c_{g} & \text { Calor específico do gás } & {\left[\mathrm{J} \mathrm{kg}^{-1} \mathrm{~K}^{-1}\right]} \\ c_{x} & \text { Calor específico do xisto } & {\left[\mathrm{J} \mathrm{kg}^{-1} \mathrm{~K}^{-1}\right]} \\ h & \text { Coef. de transferência de calor } & {\left[\mathrm{J} \mathrm{s}^{-1} \mathrm{~m}^{-2} \mathrm{~K}^{-1}\right]} \\ k & \text { Condutividade térmica } & {\left[\mathrm{J} \mathrm{s}^{-1} \mathrm{~m}^{-1} \mathrm{~K}^{-1}\right]} \\ Q_{g} & \text { Calor do gás } & {[\mathrm{J}]} \\ Q_{x} & \text { Calor do xisto } & {[\mathrm{J}]} \\ t & \text { Tempo } & {[\mathrm{s}]} \\ T_{g} & \text { Temperatura do gás } & {[\mathrm{K}]} \\ T_{g e} & \text { Temp. do gás na entrada } & {[\mathrm{K}]} \\ T_{g s} & \text { Temperatura do gás na saída } & {[\mathrm{K}]} \\ T_{\text {sup }} & \text { Temp. da superfície da partícula } & {[\mathrm{K}]} \\ T_{x e} & \text { Temperatura do xisto na entrada } & {[\mathrm{K}]} \\ T_{x s} & \text { Temperatura do xisto na saída } & {[\mathrm{K}]} \\ v & \text { Velocidade do gás } & {\left[\mathrm{m} \mathrm{s}^{-1}\right]} \\ W_{g} & \text { Vazão mássica do gás } & {\left[\mathrm{kg} \mathrm{s}^{-1}\right]} \\ W_{g e} & \text { Vazão mássica do gás na entrada } & {\left[\mathrm{kg} \mathrm{s}^{-1}\right]} \\ W_{g s} & \text { Vazão mássica do gás na saída } & {\left[\mathrm{kg} \mathrm{s}^{-1}\right]} \\ W_{x} & \text { Vazão mássica do xisto } & {\left[\mathrm{kg} \mathrm{s}^{-1}\right]} \\ W_{x e} & \text { Vazão mássica do xisto na entrada } & {\left[\mathrm{kg} \mathrm{s}^{-1}\right]} \\ W_{x s} & \text { Vazão mássica do xisto na saída } & {\left[\mathrm{kg} \mathrm{s}^{-1}\right]} \\ T_{g s} & \text { Temperatura do gás na saída } & {[\mathrm{K}]} \\ & & \end{array}$

\section{REFERÊNCIAS}

ALMEIDA, A. R. F. Investigação do mecanismo de desvolatilização de partículas de xisto. Dissertação (Mestrado em Engenharia Química) - Universidade Estadual de Campinas, 2005.

IEA (International Energy Agency). Towards a more energy efficient future: applying indicators to enhance energy policy. OECD, p. 9, Paris, França, 2009.

\section{LEVENSPIEL, O. Chemical Reaction} Engineering. New York: John Wiley \& Sons. $3^{\mathrm{a}}$ ed. p. 659. 1998.
LISBÔA, A. C. L. Investigations on oil shale particle reactions. Tese (Doutorado em Engenharia Química) - The Faculty of Graduate Studies Chemical and Bio-Resource Engineering, University of British Columbia: Vancouver, Canadá, 1997.

MELO, L. P.; LISBÔA, A. C. L. Modelagem matemática do processo de pirólise de partículas de xisto em um reator industrial. XIX Congresso Brasileiro de Engenharia Química, Búzios, Brasil, 2012.

MORATORI, C. C.; LISBÔA, A. C. L. Transferência de calor na zona de aquecimento de um leito móvel de xisto. Dissertação (Mestrado em Engenharia Química) - Universidade Estadual de Campinas, 2014.

PETROBRAS. Refinaria SIX Industrialização de Xisto. Disponível em: $<$ http://www.petrobras.com.br/pt/quemsomos/principais-operacoes/?category $=1>$. Acesso em: Fev. 2014.

SCHIESSER, W. E. The Numerical Method of Lines. Academic Press. 1991.

SCHIESSER, W. E. Computational Mathematics in Engineering and Applied Science: ODEs, DAEs and PDEs. CRC Press, Boca Ranton. 1994.

SCHÖN, J.H. Physical Properties of Rocks: Fundamentals and principles of petrophisics. Ed. Pergamon. Cap. 8. p.323378. 1996.

TONEL, G.; TAFFAREL, S. R.; NOGUEIRA, J. O. C. Processamento do xisto. Universidade Federal de Santa Maria: Departamento de Engenharia Química, Centro de Tecnologia, 2004. Disponível em: <http://www.oocities.org/br/giovanitonel/che 
mical_eng_files/process_xisto.htm>. Acesso em: Fev. 2014. 